of course the first. Fields such as materials and microelectronics have followed more recently.

Currents developments show a rapid build up of more and more dedicated institutes at universities. These institutes are not necessarily supported by FOM; nor are they shared exclusively by physicists. Formal cooperation between physicists and scientists from other disciplines at the national level can be identified in surface science and materials: for example, with chemists at the Debye Institute in Utrecht, at the Materials Study Centre in Groningen and in the FOM-ALMOS collaboration between FOM and the Universities of Amsterdam and Leiden. But there is also cooperation in mathematical physics (with mathematicians), computational physics (with computer scientists) and high resolution electron microscopy (with scientists from several disciplines at the recently established HREM Centre in Delft).

With encouragement from the government, the three technical universities have agreed on complementary research programmes in the fields of semiconductors and microelectronics and one outcome has been the formation of the Delft Institute for Microelectronics and Submicron Physics (DIMES). A

\title{
Electron Wave Function Measured
}

In 1988 scientists at AMOLF realized the dream of every atomic physicist. Using a laser belonging to the University of Amsterdam they actually measured the "classi$\mathrm{cal}^{\prime \prime}$ orbit time of the radial motion of a Rydberg electron around the atomic core. The key to the experiment was to excite the electron by a short laser pulse at the $n=19$ manifold of rubidium, thus forming a dispersion free wavepacket at time $t=0$. With a second pulse, the delay time of which can be adjusted, the atom is then ionized. Interaction with the laser field is only possible when the electron is close to the core. Peaks in the ionization yield occur if the delay is a multiple integral of the "classical" Bohr orbit time. This experiment directly demonstrates the link between classical and quantum mechanics.

The figure plots the observed probability of finding an electron close to the atomic core as a function of the time between the initial excitation laser pulse and the subsequent ionisation pulse. For this experiment, the complete $n=19$ manifold of rubidium was excited in a $645 \mathrm{~V} / \mathrm{cm}$ electric field. Successive peaks arise when the delay time is a multiple of the electron's orbit time around the nucleus.

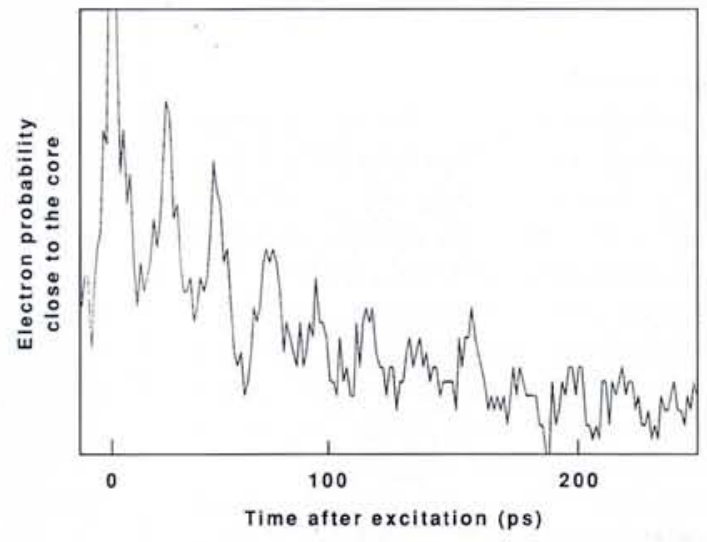

Institute for Atomic and Molecular Physics, Amsterdam

\section{Grenoble - Rhône-Alpes - France}

The European Synchrotron Radiation Facility is constructing a state of the art storage ring for $6 \mathrm{GeV}$ electrons and/or positrons to be operated as a high brilliance synchrotron radiation source in the field of $X$-ray from 1994 on. Financing of the ESRF is shared by 11 European countries.

\section{EUROPEAN SYNCHROTRON RADIATION FACILITY}

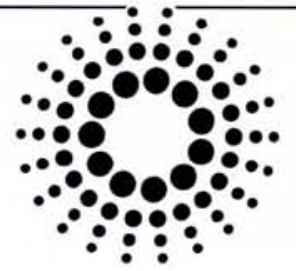

In the present build up phase, the European Synchrotron Radiation Facility offers you interesting career prospects and a unique opportunity to develop and apply your skills in an international hi-tech environment in fields as varied as

Materials Sciences: diffraction, elastic diffuse scattering, inelastic scattering, extended X-ray absorption fine structure.

condensed matter theory, magnetic scattering.

X-ray Beam Lines: design, imaging, optics, detectors.

Physics,

Mechanics,

Ultra High Vacuum,

Power Supplies,

Applied Computer Science: hardware \& Software

Chemistry,

Magnets: undulators $\mathcal{E}$ wigglers

Hydraulics and Cooling.

Radio frequency.

Electronics,

Remote Control \& Operation of Complex Machines. Biology.

We recruit now:

\section{Scientists*, Engineers and Technicians}

*Scientists are generally on fixed-term contracts (max. 5 years)

who give evidence of relevant education, and who have experience in research institutes, universities or high-tech industries. Candidates with at least some years of experience in a similar situation, or with a professional background combining education and/or experience from one or more of the fields mentioned above are particularly invited to apply
The working language is English:- knowledge of French is desirable.

For more information about the ESRF, employment conditions and specific vacancies, you can give your name, address and field of professional experience directly to us in Grenoble, and we shall provide you with the information needed. and an "Application Form".

The completed "Application Form" must reach us at thellatest on 15 September 1990.

ESRF (Recruitment Officer), Boîte Postale 220, F-38043 Grenoble Cedex.

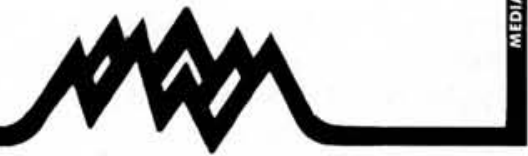

